\title{
A Curve-Skeleton Extraction Algorithm Based On Vector Fields for 3D Model Retrieval
}

\author{
Sun Ting ${ }^{1,2}$,Zhou Wen-Gang ${ }^{1}$ and Geng Guo-Hua ${ }^{2}$ \\ ${ }^{1}$ College of Computer Science and Technology, Zhoukou Normal University, \\ Zhoukou 466000, China \\ ${ }^{2}$ Institute of Visualization Technology, Northwest University, Xi'an 710069, China \\ 49637346@qq.com
}

\begin{abstract}
Feature extraction is a key issue for $3 d$ model retrieval. A novel architecture to extract the curve-skeleton of $3 d$ model is introduced. The algorithm firstly calculates the $3 D$ vector fields of models represented by discrete unit, and then extracts the hierarchical curve-skeleton based on topological features of the critical curve and critical points of the vector fields. The similarity among 3D curve-skeletons is measured by using an improved Earth Mover's Distance (EMD) algorithm. The curve-skeleton extracted with this novel algorithm can be used to categorize models and implement global matching and partial matching.
\end{abstract}

Keywords: 3d Model Retrieval; Feature Extraction; Curve-Skeleton

\section{Summary}

How to introduce the local characteristic information to the description of $3 \mathrm{D}$ model feature, to realize the $3 \mathrm{~d}$ model fine retrieval which based on the model components, is a further research problem in the area of $3 \mathrm{~d}$ model retrieval ${ }^{[1]}$. 3D skeleton information can control the level of detail very well, express the shape feature of the model truly and directly, not only can the model do the global matching, but also can do the local matching, to realize the representation and retrieval of $3 \mathrm{~d}$ model multi hierarchy ${ }^{[2,3]}$. At present, the extraction method of $3 \mathrm{~d}$ framework which proposed by scholars at home and abroad mainly have two kinds: a kind of method is based on the elaboration ${ }^{[4,5]}$, however this kind of algorithm is very sensitive to noise, small disturbance can lead to wrong skeleton extraction, it is difficult to guarantee the accuracy and smoothness of the skeleton, which needs for further adjustment ${ }^{[6]}$. Another kind of algorithm is based on the distance transform ${ }^{[7-11]}$, the skeleton point position of this kind of algorithm is more accurate, But when the object branch is tiny, it is difficult to guarantee the connectivity of the whole skeleton ${ }^{[7,9]}$ meanwhile, according to the above definition, the curve skeleton of some objects is an isolated point, this framework not only difficult to used for the design of 3D animation, nor to describe the topological structure objects ${ }^{[8,11]}$.

This paper presents an approach to extract the multi-scale curve skeleton of 3D model which based on the vector field. Firstly, calculate the vector field of 3D model represented 
of discrete voxel, the point whose size of the components of vector is zero as the key point, according to the vector search method to connect each key point, to get the nuclear skeleton of objects; Then select the boundary point who has the large curvature to continue growth skeleton to obtain the hierarchical skeleton of objects. By means of the improved EMD method to measure the similarity of linear skeletons. The experimental results show that the method has the low time complexity, the strong versatility and robustness, the curve skeleton extracted based on this algorithm can achieve the model classification, global matching and partial matching, can improve the retrieval efficiency effectively.

\section{Extraction Method of Multi-Scale Curve Skeleton}

\subsection{The Main Idea and the Procedure Of The Algorithm}

Assume that there are the same charge which uniform distribution on the edge of the object, these charges are formed a stable electric field inside the object, it is a vector field, acting the point whose field strength is zero as the key point, according the vector search method ${ }^{[12]}$ to connect each key point, to get the object nuclear skeleton; Then select the boundary point who has the large curvature to continue grow the framework, thus get the object of multi-level framework. The specific algorithm is as follows:

Step 1 calculate the vector field over a discretization of the entire 3D object;

Step 2 determine the key point in the vector field;

Step 3 based on the following three kinds of seed point, using the force tracking algorithm to extract the multi-scale curve skeleton;

Step3.1 (Level 0) : use key point as the seed point, to generate the core skeleton, that is ,the zeroth layer of the hierarchical curve skeletons;

Step3.2 (Level 1): use the low divergence voxel as the seed point, to get the higher complexity of the skeleton, that is, the first layer of the hierarchical curve skeletons;

Step3.3 (Level 2) : use the seed point which selected by user, to get the more higher complexity of the skeleton, that is, the second layer of the hierarchical curve skeletons;

In Step 1, we compute a vector field over a discretization of the 3D object. If the given 3D model is of non voxel representation, then it is need for the voxelization process. Set $O \in Z^{3}$ is a $3 \mathrm{~d}$ voxel grid model, and $v f: Z^{3} \rightarrow R^{3}$ is a vector function, used to calculate the vector for each grid voxel, $\vec{v}=v f(p)$ the vector field of $3 \mathrm{D}$ models composed of the vectors at the voxel model (or its subset). Once a vector field is computes, in Step 2, we locate the critical points of this vector field. It is the points whose size of components is zero, that is, $v f(p)=0$. In Steps 3 we compute the divergence of the vector field, at each voxel. The divergence measures the "sinkness" of a position in the vector field. Then, extract the curve skeleton of 3D models step by step, generate a complexity increasing curve skeleton set. 


\subsection{Determine the Critical Point}

The critical point is the point whose size of the components of vector is zero in the vector field. Set $P=(x, y, z)^{T}$ is the center of the minimum candidate unit, $F=f v(P)$ is the vector value at point $P . J(P)$ is the Jacobian matrix at $P$ :

$$
J(P)=\left|\begin{array}{ccc}
\frac{\partial f v_{x}}{\partial x} & \frac{\partial f v_{x}}{\partial y} & \frac{\partial f v_{x}}{\partial z}
\end{array}\right|
$$

Among them, $f v_{x}, f v_{y}$, and $f v_{z}$, are the components $(x, y, z)$ of the vector field function $f v$ respectively, we solve the following equation: $J(P) \cdot d P=-F$ for $d P$, to determine the position $P=P+d P$ of next guess according to the value of $A$.

Repeat the above operation, till $F=f v(P)$ is zero, or the next guess position is not in the initial candidate voxel unit.

The real part of the eigenvalue of Jacobian matrix at the key point $P$ is positive ,which indicates the presence of a repulsion direction(the specific direction is determined by the corresponding feature vector); The real part of the eigenvalue is negative value, which indicates the presence of attracting direction; The imaginary part describes the helical motion around the corresponding points. According to the real and imaginary parts of the eigenvalues, the key points can be divided into: the attracting point the repulsion point and the saddle point. If the sign of all the real parts of the eigenvalues are the same sign, then the key point is the attracting point (is negative) or repulsive point (is positive). If the two real part are of the same number, and another has the opposite sign, then , the critical point is a saddle point. The vector point to the saddle point along the negative eigenvalue direction (the specific direction is determined by the eigenvector direction of Jacobian matrices), along the positive eigenvalue direction deviates from the saddle point.

\subsection{Vector Search Algorithm}

The vector search algorithm ${ }^{[12]}$ determine a starting point in different positions of vector fields, use the simple Euler integration method ,extract the curve segment which constitute the curve skeleton. The specific algorithm is as follows:

Step $1 \quad P$ is the initial position (seed point), $C=\{P\}$ is the linear skeleton segments which Has been calibrated ;

Step 2 To estimate the vector field at point $A$;

Step 3 If $F \neq 0$ and point $P$ hasn't been visited, then the point $P$ is the next position in the direction of $F, C=C \cup\{P\}$;

Step 4 estimate the vector field value at the new position : $F=f v(P)$.

When implement the algorithm, act the seed point as the starting position, using the three line interpolation to estimate the vector field value $F: F=f v(P)$ of any position at $P$. If the vector field value of current position is zero, then it shows that the position is the 
key point, the algorithm terminates. Otherwise, moved forward along the direction pointed by $F$, the next location is:

$$
P=(2),+\operatorname{stepSize} \cdot \frac{\vec{F}}{\|\vec{F}\|}
$$

In order to avoid computing the vector outside the discrete grid, make a appoint that when point $P$ in the model of external or boundary $f v(P)=0$, the algorithm was terminated.

\subsection{Multi-Level Linear Skeleton Extraction}

According to the clockwise order in turn with the key point, low divergence and high curvature points as the starting position, using the vector search algorithm, extracting multi-scale linear skeleton of increasing complexity from the vector field. Firstly, act the key point in the vector field as the seed point, the most basic skeleton can be obtained(the core framework), constitute the zeroth layer hierarchical curve skeletons; Secondly, act the low divergence point as the seed point, increase the number of curve segments in the core skeleton, obtain the higher layer of the linear skeleton, constitute the first layer hierarchical curve skeletons; Finally, act the high curvature points as the seed points, Increase the curve segments in the front stage framework, obtain the curve skeleton of a higher level, constitute the second layer hierarchical curve skeletons. The specific algorithm is as follows:

Step 1 Step 1 To extract the core framework based on key point

The core skeleton based on key point is the zeroth layer of the 3D model of multilevel linear framework: integrate forward or backward along The characteristic direction of the key point, extracte the Integral curve of vector field. The core framework is a subset of integral curve which connect all the key points in the vector field, the curve segments which constitute the core framework are all from the beginning of a key point, to the end of another key point.

The forward integration method is the search along the characteristic direction of every initial critical point,that is: $P=P+\operatorname{stepSize} \cdot \frac{\vec{F}}{\|\vec{F}\|}$, get to another key point, the curve segments are formed is the core skeleton segments; The backward integration method is the search along the characteristic opposite direction of every initial critical point, that is: $P=P+\operatorname{stepSize} \cdot \frac{\vec{F}}{\|\vec{F}\|}$, get to another key point, also can form the core framework segments.

Step 2 Extract the linear skeleton section based on low divergence point

Curve section of low divergence point is the first layer of the 3D model of multilevel linear framework. Divergence of vector field is a scalar quantity, which can be used for representing the degree of divergence of vector field of each point in space , in physics, the meaning of divergence is field of source. When $\operatorname{div} F>0$, it shows that the point has the source of emission flux(divergent source); When $\operatorname{div} F<0$, it shows that the point has the negative source of absorb the flux(hole or sink); When 
$\operatorname{div} F=0$,it shows that the point has no source. Which can use the formula (3) to calculate the divergence of specified point:

$$
\operatorname{div} F=\nabla F=\frac{\partial F_{1}}{\partial x}+\frac{\partial F_{2}}{\partial y}+\frac{\partial F_{3}}{\partial z}
$$

Among them, $F_{1}, F_{2}, F_{3}$ are the components of vector $F$ in the direction of $(x, y, z)$ axis respectively.

The low value of divergence describes the "concavity" of a point, based on the principle of "local minimum", select the voxels whose divergence value is below a threshold value or less than all its adjacent voxels as the new seed point. Act each new seed point as the starting point, use the vector search algorithm to determine a curve. By changing the dispersion threshold constantly, users can select different seed points, thus, form the first layer of a linear skeleton. The linear skeleton based on the divergence values of $t$ is a skeleton subset of all the generated skeleton large than $t$. When meeting the access point, the vector search algorithm o terminates, the new generation of curve segments connected to the existing framework.

Step 3 Extract the skeleton section based on the seed points of high curvature

Skeleton segments of the seed points of high curvature are the second layer of 3D model of multi hierarchical linear skeleton. Another class of seed point set which Used to generate the skeleton curve is composed of 3D objects lobe, base on these seed point will generate the curve section ${ }^{[13]}$ from the model surface to the existing skeleton. If the model is not convex, then calculate the boundary curvature ${ }^{[14]}$, based on the local maximum criterion, act the curvature among it that greater than a certain threshold as the boundary seed point.

The disadvantage that select seed point based on curvature of the boundary is the size of the curvature is susceptible to interference of boundary noise mode, method to solve this kind of problems is that: when calculate the curvature of the boundary voxels, should not only consider the surface, the boundary and the adjacent points, but also consider the extended adjacent voxel unit ${ }^{[14]}$.

The skeleton curve segments starting from the boundary seed points form the second layers of 3D model of multi-level linear skeleton, the curve segments of this layer will be connected to the first layer framework.

\subsection{The Algorithm Flow}

Step 1 Demarcate the 3D model of the boundary voxels as the vector field source; Step 2 Calculate the vector function of each voxel model, to get a 3D vector field; 


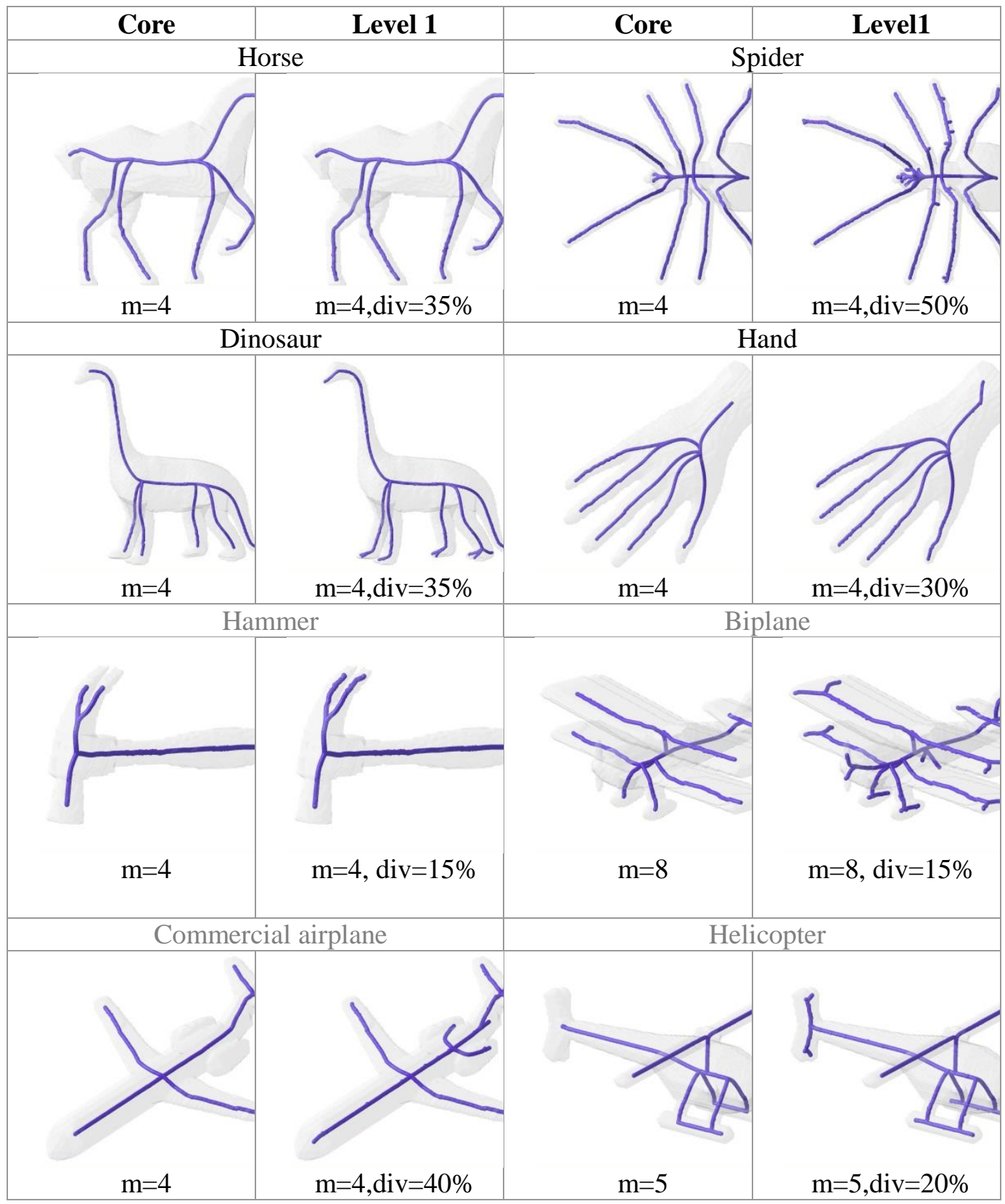

Figure 1. Curve-Skeletons of Several Objects. (A) Core Skeleton, (B) Level 1 Curve-Skeleton. The Parameters Are Given Next To Each Curve-Skeleton

Step 3 Generate core skeleton: detect the key point of the vector field, use the path of integration in vector field to connect these key points, generate the nuclear skeleton;

Step 4 Generate the first layers of skeleton: calculate the divergence of each voxel, choose low divergence point as the new seed point, generate a new skeleton curve segment; Change the divergence threshold ,generate a skeleton layer that connect to the nuclear skeleton, constitute the first layer hierarchical curve skeletons;

Step 5 Generate the second layers of skeleton: Calculate the curvature of each boundary voxels, selecte the new seed point based on curvature threshold set by the users, generate a new skeleton which connect the nuclear skeleton to the first layers of skeleton, constitute the second layer hierarchical curve skeletons. 


\section{The Algorithm Analysis and Experiment212}

This section designed two groups of experiments to test the validity of the method for extracting and matching of linear skeleton which proposed from the paper.

\subsection{Time Complexity}

To computate the time complexity of a 3D model vector field depends on the body prime $N_{a}$ constructed this model and the boundary body prime $N_{a}$, that is, $O\left(N_{a} \times N_{b}\right)$, because $N_{b}$ is a part of $N_{a}$, therefore, its time complexity is close to $O\left(N_{a}^{2}\right)$. While in the computation of the whole linear model framework, the calculation of the vector field is the most time consuming, accounting for about $98 \%$ of total time.

And for normal divergence field, the calculation methods are as follows: set $O S$ is the boundary voxel model set, $O I$ is the internal model element set, then the whole model is $O=O S \cup O I . c(v)$ is the counter corresponding to model voxels $v$.

Which can be seen from the above algorithm, each voxel in the model, insert and delete from the sequence only for one time, when the sequence is empty the algorithm terminates. Obviously, the time complexity of calculation to the normal field emission model is $O(n)$, where $n$ is the model body prime.

\subsection{The Classification and Retrieval Of 3D Model Library}

First of all, centralized the 1081 model from the training set and test database of Princeton (Princeton Shape Benchmark Database, PSB) ${ }^{[15]}$ as the experimental data sets, the data set was divided into 99 non empty classes ${ }^{[16]}$. In the experiment, firstly, extract the curve skeleton of every model in database ,then using the improved EMD method, to calculate the bulldozer distance EMD of each model between data set and other model. If the model has been classified, then the system returns a similar model and query. In the experiment, only classify the correct match, based on the statistics of all the matches, the optimal matching and the query based on the algorithm in this paper belong to the same category accounted for $71.1 \%$; With the query of the same parent class accounted for $74.3 \%$.

In the second experiment, detection system in the return of the former $\mathrm{T}$ matching model, how many models have the same kind as the query. Figure 2 shows the effect of retrieval model based on line below Ieval results and the similarity according to the query in descending order, display and query the most similar ten top model. Below each results of the model image indicates the model and the EMD of query model. 


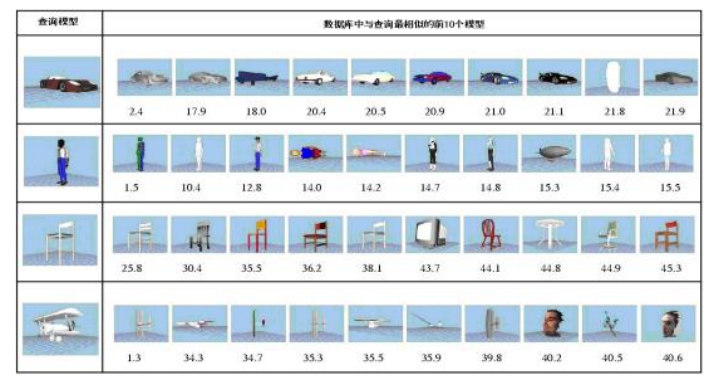

Figure 2: The Retrieval Results Based On Curve-Skeleton

The use of EMD algorithm can obtain query the corresponding between all models in database, but it is difficult to determine the corresponding area of the two linear skeleton whether represent the components of the similar model, it needs to have the priori knowledge corresponding semantic of the model components. While this algorithm can make the model group matching visualization, easy to identify the matching model components, the users can determine whether it is meaningful to the corresponding model components quickly. Compared with the skeleton extraction method proposed by reference $^{[10-17]}$, the skeletonization method based on vector field has stronger robustness to the noise of model boundary.

Certainly, using this algorithm can not only determine the linear framework such as a thin disc or mushroom shape model. The linear skeleton matching method which based on EMD can find the loose relationship between skeleton points in the process of skeletonization, to compensate for the lack of linear skeleton.

\section{Conclusion}

Extracting the simple feature of 3D model, converting the similarity matching between 3D model to the comparison between corresponding characteristics is the basic train of thought to realize the 3D model retrieval. In this paper, proposed the 3D model of linear skeleton extraction framework, the framework first computes a vector field of 3D model which represent of the discrete voxel, using the results of vector field's topology characteristics of key points, key curve etc, extract the linear skeleton; Then with the aid of EMD bulldozer to measure the similarity of linear skeletons to Realize the retrieval of 3D model .The experimental results show that the framework has the low time complexity, the strong versatility and robustness; The curve skeleton extracted based on this algorithm can achieve the classification and retrieval of model, and the retrieval effect is better.

\section{Acknowledgment}

This work was supported by the National Key Basic Research Development Program (973 Program) preliminary study of special (2011CB311802), Science and Technology Development Plan of HeNan province in China(Grant122400450356, 142400411058), Province Soft Science and Technology Development Plan Project of Henan in China(132400410927). 


\section{References}

[1] Liu Zhen-Bao, Bu Shu-Hui and Kun Zhoul. A Survey on Partial Retrieval of 3D Shapes[J].Journal of Computer Science and Technology, 2013, 28(5): 836-851.

[2] CHEN Jun, WANG Bo and ZHOU Yu.. Shape Recognition and Classification Based on Skeleton Junction Nodes Features [J]. Computer Science, 2011, 38(1).

[3] LIN Jiao,LI Zhong and JIN Xiao-gang. Skeleton Extraction Method Based on Convex Hull and OBB[J].Journal of Computer-Aided Design \& Computer Graphics， 2013，24(6).

[4] C. M. Ma and S. Y. Wan. Parallel thinning algorithms on 3D binary images [J]. Computer Vision and Image Understanding, 2000, 80(3): 364-378.

[5] W. Xie, R. P. Thompson and R. Perucchio. A topology-preserving parallel 3D thinning algorithm for extracting the curve skeleton [J]. Pattern Recognition, 2003, 36(7): 1529-1544.

[6] CHE Wu-Jun, YANG Xun-Nian and WANG Guo-Zhao. A Dynamic Approach to Skeletonization[J].Journal of Software, 2003,14(4):818-823.

[7] W.P. Choi, K. M. Lam and W. C. Siu. Extraction of the Euclidean skeleton based on a connectivity criterion[J]. Pattern Recognition, 2003, 36(3): 721-729.

[8] DING Yi, LIU Wen-Yu and ZHENG Yu-Hua. Hierarchical Connected Skeletonization Algorithm Based on Distance Transform [J]. Journal Infrared Millimeter and Waves 2005, 24(4): 281-285.

[9] LIU Jun-Tao, LIU Wen-Yu and WU Cai-Hua. A New Method of Extracting Objects' Curve-skeleton [J].Acta Automatica Sinica, 2008(6), and 22(11): 1241一1251.

[10] N. D. Cornea, D. Silver, X. S. Yuan and R. Balasubramanian..Computing hierarchical curve-skeletons of 3D objects [J]. The Visual Computer, 2005, 21(11): 944-955.

[11] SHEN Wei, BAI Xiang and YANG Xing-Wei. Skeleton pruning as trade-off between skeleton simplicity and reconstruction error [J]. Sci China Inf Sci, 2013, 56: 048101(14), doi: 10.1007/s11432-012 -4715-3.

[12] Chuang J. H., Tsai C. H. and Ko M. C. Skeletonization of Three-Dimensional Object Using Generalized Potential Field [J]. IEEE Transactions on Pattern Analysis and Machine Intelligence, 2000 22(11): 12411251.

[13] Abdel-Hamid G. and Yang Y..Multiresolution Skeletonization: An Electrostatic Field-Based Approach [J].ICIP (1) 1994, pp.949-953.

[14] Hameiri E. and Shimshoni I. Estimation the Principal Curvatures and the Darboux Frame from Read 3D Range Data[C]. 1st International Symosium on 3D Data Processing Visualization and Transmission, 2002 6:258-267.

[15] Princeton Shape Retrieval and Analysis, 3D Model Search. http://shape.cs.princeton.edu/search.html.

[16] Shilane P., Min P. and Kazhdan M. The Princeton shape benchmark. Shape Modeling International, June 2004.

[17] Kazhdan M., Funkhouser T. and Rusinkiewicz S. Rotation invariant spherical harmonic representation of 3d shape descriptors[C] Eurographics Symposium on Geometry Processing, pp.167-175,2003. 


\section{Authors}

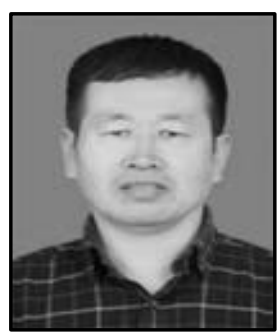

Sun Ting was born in HeNan, China, on June 20, 1972. He received the M.S. degree in Computer Software and Theory from Zheng Zhou University and The PHD in Computer Software and Theory from Northwestern University in 2002 and 2011respectively.His research interests include digital image processing, Cloud computing and Internet of Things.

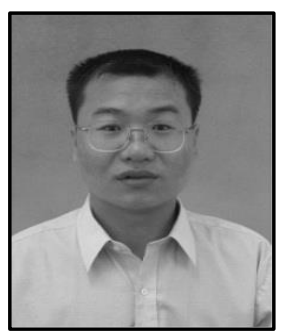

Zhou Wen-Gang received B.Eng Degree in computational mathematics from HeNan Normal University and the M.Eng Degree in computer application technology from North China University of technology in 1997 and 2007 respectively, He is currently researching on the analysis and design of Intelligent algorithm.

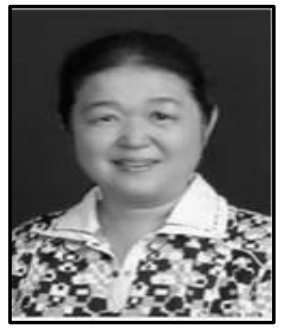

Geng Guohua was born in 1955. Ph.D., professor, Ph.D. supervisor, her research interests include. Intelligent information processing, Database and knowledge base. 